\title{
On the response of fading times of upper homosphere radar echoes to solar and geomagnetic disturbances
}

\author{
C. M. Hall ${ }^{1}$, S. Nozawa ${ }^{2}$, C. E. Meek ${ }^{3}$, and A. H. Manson ${ }^{3}$ \\ ${ }^{1}$ Tromsø Geophysical Observatory, Tromsø, N-9037 Norway \\ ${ }^{2}$ Nagoya University, 464-01 Japan \\ ${ }^{3}$ University of Saskatchewan, S7N 5 E2 Canada
}

(Received March 22, 2001; Revised January 4, 2002; Accepted April 11, 2002)

Four years of auroral latitude radar echo signal fading time data from the Tromsø MF radar compared with geomagnetic and solar indices yield strong evidence to suggest, although not prove, that upper mesospheric turbulence may be enhanced during periods of geomagnetic activity. We present a critique of hypotheses to explain such effects.

\section{Introduction}

Several instances of indications that mesospheric dynamics is influenced by solar or geomagnetic effects have been published in recent years. Danilov et al. (1979) presented measurements of the turbopause altitude over Heiss Island $\left(80^{\circ} \mathrm{N}, 58^{\circ} \mathrm{E}\right)$ derived from a series of rocket soundings. These authors did not attempt to explain their findings, but one might surmise that turbulence is being enhanced at or just below the "usual" turbopause causing dominance of turbulent mixing over molecular dissipation to extent to slightly higher altitude. That ion-drag might augment or even induce dynamic instability in the neutral flow at such altitudes has been proposed by Hall (2001a), and to some extent demonstrated to be a feasible hypothesis by Hall and Aso (2000). Presumably, other mechanisms are responsible for the weak correlations between solar and/or geomagnetic indices and other scales of dynamics such as tides (Hocke and Igarashi, 1998) and the newly observed quasi 27-day oscillation in the MLT wind (Luo et al., 2000). For these longer scales of dynamics, it is more likely that an enhanced solar UV flux heats the stratosphere and that the resulting change in temperature gradient with height affects the gravity wave energy and momentum fluxes into the mesosphere. Elements of this have already been studied at Saskatoon $\left(57^{\circ} \mathrm{N}, 107^{\circ} \mathrm{W}\right)$ in comparing gravity wave band intensities with solar flux and the $K p$ index (Gavrilov et al., 1995) and even earlier by Manson and Meek (1986) who noted the coincidence of gravity wave intensity and $K p$ maxima.

Even so, one might expect changes in gravity wave breaking and a corresponding signature in turbulence intensities. As an example, a higher stratosphere temperature might give rise to a greater lapse rate in the lower mesosphere, which, in turn, would give more opportunity for gravity wave breakdown and therefore increased turbulent energy dissipation

Copy right (C) The Society of Geomagnetism and Earth, Planetary and Space Sciences (SGEPSS); The Seismological Society of Japan; The Volcanological Society of Japan; The Geodetic Society of Japan; The Japanese Society for Planetary Sciences. rates. In the same scenario, gravity waves would lose energy before propagating to the mesopause region where, consequentially, the energy dissipation would be reduced.

In this study, advantage will be taken of the four-year long time-series of radar echo signal fading times, $\tau_{1 / 2}$, currently available from the Tromsø MF radar at $69^{\circ} \mathrm{N} 19^{\circ} \mathrm{E}$. This radar, jointly owned by University of Tromsø (Norway), University of Saskatchewan (Canada) and Nagoya University (Japan) has been described in detail by Hall (2001b); all necessary references to system parameters may be found therein. Radar echoes are received on three spaced antennae and the so-called "Full Correlation Analysis (FCA)" (Meek, 1980; Briggs, 1984) is applied to the signals yielding horizontal velocity and signal fading times at heights from 50 to $140 \mathrm{~km}$ and with time resolutions of 2 or 5 minutes. For the purposes of this study, we shall convert the measured fading time (a "radar parameter"), $\tau_{1 / 2}$, to an equivalent geophysical parameter, $\epsilon^{\prime}$, having the dimensions of energy dissipation rate. The noise reduction algorithm of Meek (1990) is applied to the signal fading times prior to calculation of $\epsilon^{\prime}$. Then, from these fading times, a turbulent velocity is estimated: Holdsworth et al. (2001) address turbulent velocity estimation and thoroughly explain the assumptions. The absolute values of energy dissipation rate are then obtained from the velocities by the method described by Hocking (1999). In the mesosphere, $\epsilon^{\prime}$ may be interpreted as an upper limit for the turbulent energy dissipation rate, $\epsilon$ since in this height regime it can be argued that the structures responsible for the radar backscatter are made indistinct by a combination of perturbation of velocity by turbulence and turbulent dissipation of the structures themselves. In the thermosphere, where viscosity damps out turbulent motion, it is not valid to interpret $\epsilon^{\prime}$ as a metric for turbulent intensity. As we shall see, however, it is interesting to represent $\tau_{1 / 2}$ as $\epsilon^{\prime}$, provided we bear in mind the above caveat. Recall also that a reduction in $\tau_{1 / 2}$ is brought about by an increase in the effectiveness of perturbing the scatterers responsible for the radar echo. Finally, for the purposes of this study, 

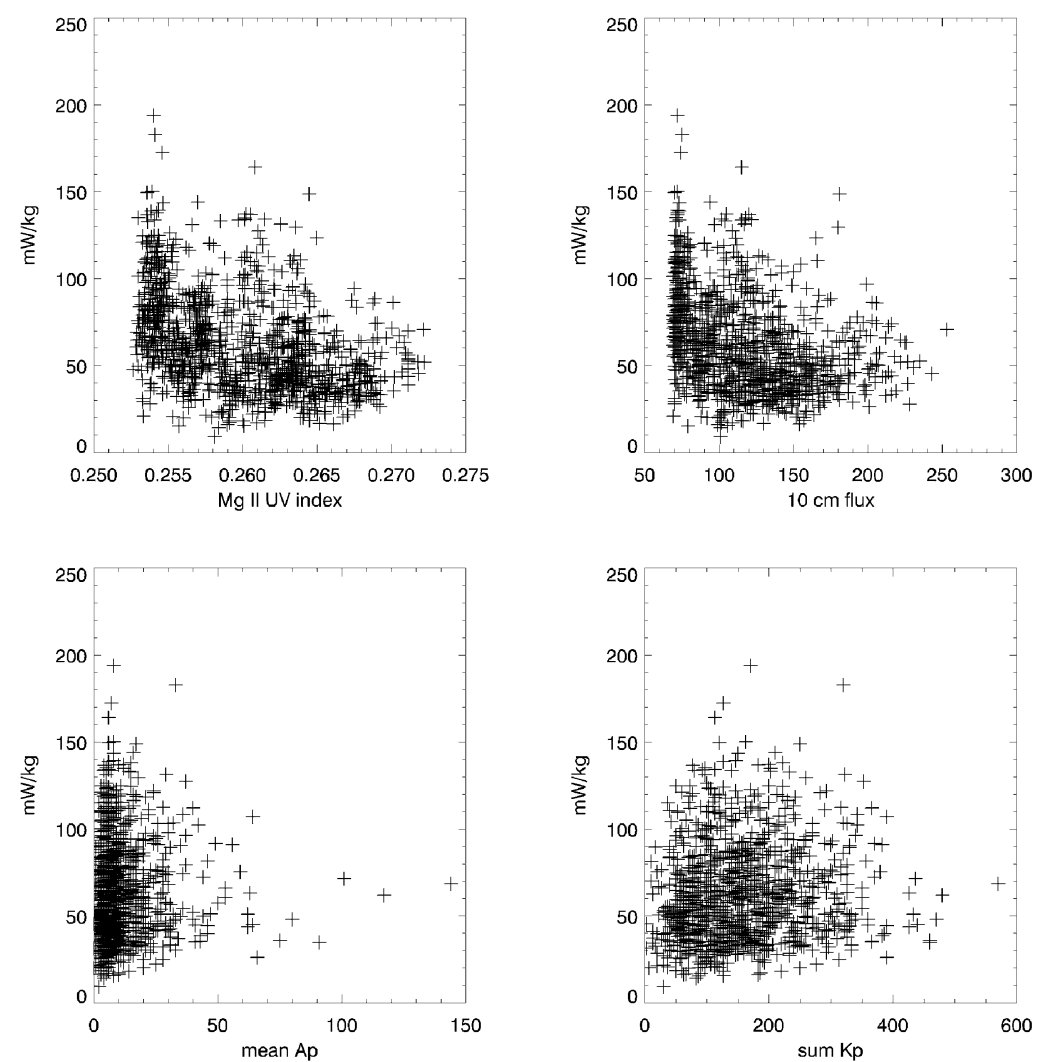

Fig. 1. Scatter plots of solar and geomagnetic parameters versus upper limit for turbulent energy dissipation rate, $\epsilon^{\prime}$ at $100 \mathrm{~km}$ altitude. Day averages of signal fading time expressed as $\epsilon^{\prime}$ (in $\mathrm{mWkg}^{-1}$ ) are used for 1997-2000 inclusive. Top left: UV flux index; top right: $10.7 \mathrm{~cm}$ flux; bottom left: mean $A p$; bottom right $\Sigma K p$.
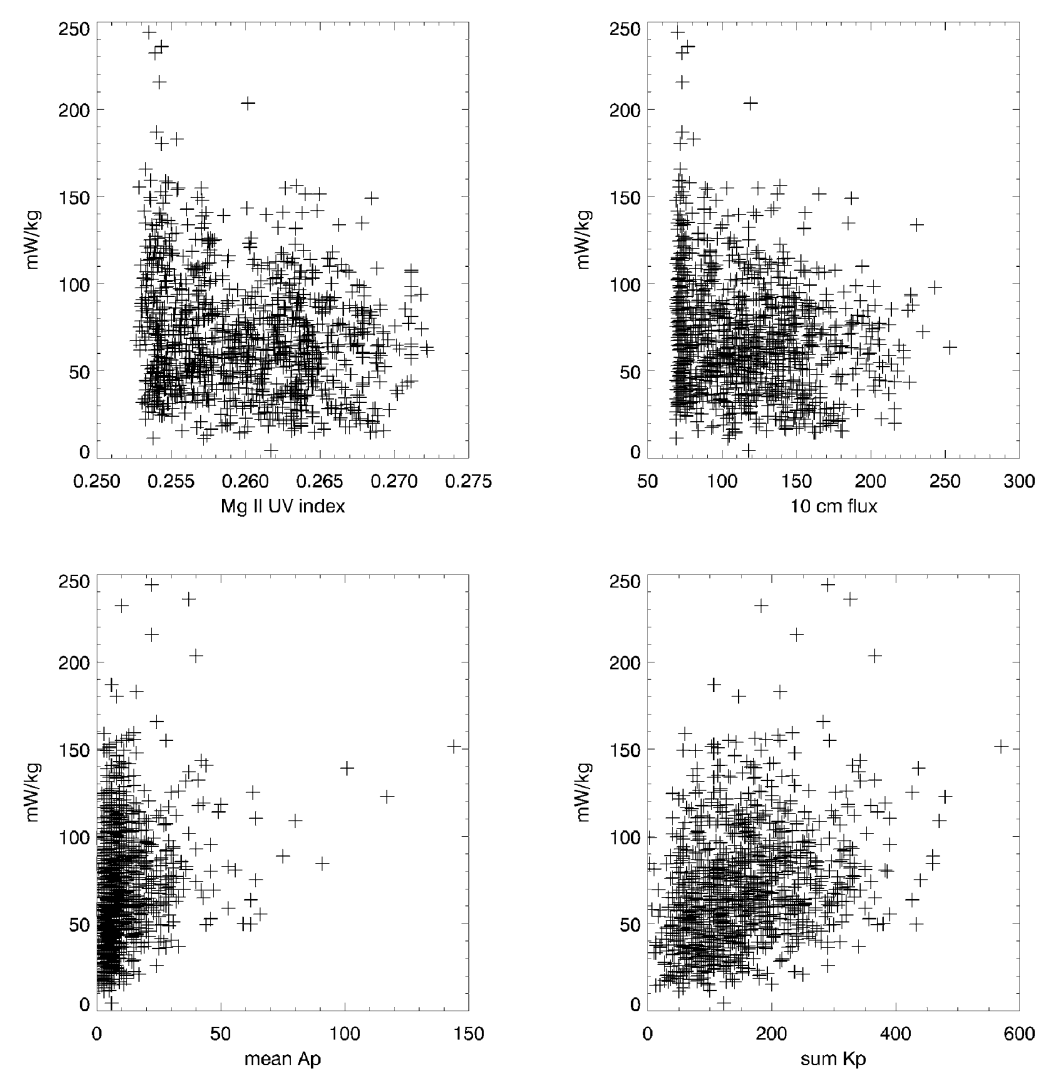

Fig. 2. As for Fig. 1 but at $115 \mathrm{~km}$ altitude. 
day averages were obtained, starting 1st January 1997 and ending 31st December 2000.

\section{Exploratory Comparisons with Solar and Geo- magnetic Parameters}

In order to facilitate filtering, the time series of $\epsilon^{\prime} \mathrm{s}$ was interpolated onto a regular grid of one-day spacing. Since it is unusual for data to be completely absent for a given day, it was not felt that any significant bias would be introduced.

For each day in the four-year period 1997-2000 inclusive, the $10.7 \mathrm{~cm}$ flux and the mean $A p$ and $\Sigma K p$ indices were obtained from the World Data Centre. In addition, $\mathrm{Mg}$ II UV indices [http://wwwsolar.nrl.navy.mil/ susim_uars_mgii_index.html] UARS data were obtained. These parameters were selected following the lead of Luo et al. (2000). Rather than attempt cross-correlations, we have simply chosen to make scatter plots. Time series of $\epsilon^{\prime}$ at several altitudes were selected: only the results for $100 \mathrm{~km}$ and $115 \mathrm{~km}$ are shown here (Figs. 1 and 2 respectively). Before examining these comparisons, the underlying physics should be considered. Turbulence is primarily instigated by convective and dynamic instabilities in motions that are in turn driven by the interplay between solar heating and the earth's rotation. Fluctuations in parameters such as those described here represent very small perturbations in this driving. The proportion of variability in turbulent energy dissipation due to these perturbations cannot be expected to be very significant in relation to other sources, such as changing gravity wave flux due to tropospheric weather systems and the general mesospheric meteorology. Also, above the turbopause, unknown mechanisms (presumably a combination of molecular diffusion and electrodynamics) are responsible for perturbing and destroying the scattering structures.

Let us now consider Figs. 1 and 2 bearing in mind that we expect a considerable spread in $\epsilon^{\prime}$ values. Very little indication of correlation between $\epsilon^{\prime}$ and any parameter is evident. However, it is possible that a weak positive correlation exists with $\Sigma K p$. Similarly a weak anticorrelation with the UV index might be discerned; recall that Luo et al. (2000) proposed that stratospheric heating due to UV absorption somehow affected the quasi 27-day oscillation amplitude. The coupling to $K p$, moreover, supports the hypothesis of Hall (2000a) and we shall explore this further.

\section{Comparisons with Local $k$ Indices}

Encouraged somewhat by the hint of a correlation with $\Sigma K p$, and considering that any ion-drag destabilisation of the neutral flow is likely to be a very local phenomenon, we obtained values of the three-hour $k$ index for the entire four-year period under study from the University of Tromsø magnetometer nearest the MF radar (approximately $15 \mathrm{~km}$ distant). Figure 3 shows scatter plots of $\epsilon^{\prime}$ versus $\Sigma k$ for a selection of heights. Although the spread is large, a trend towards a grouping can be seen. Qualitatively, the greatest evidence of a correlation appears to be at or around $115 \mathrm{~km}$. It could be anticipated that correlation would be most pronounced around such altitudes: below the unperturbed turbopause, turbulent generation mechanisms not related to geomagnetism (gravity wave breaking etc.) are present, while neutral viscosity increases rapidly with altitude thus damp-
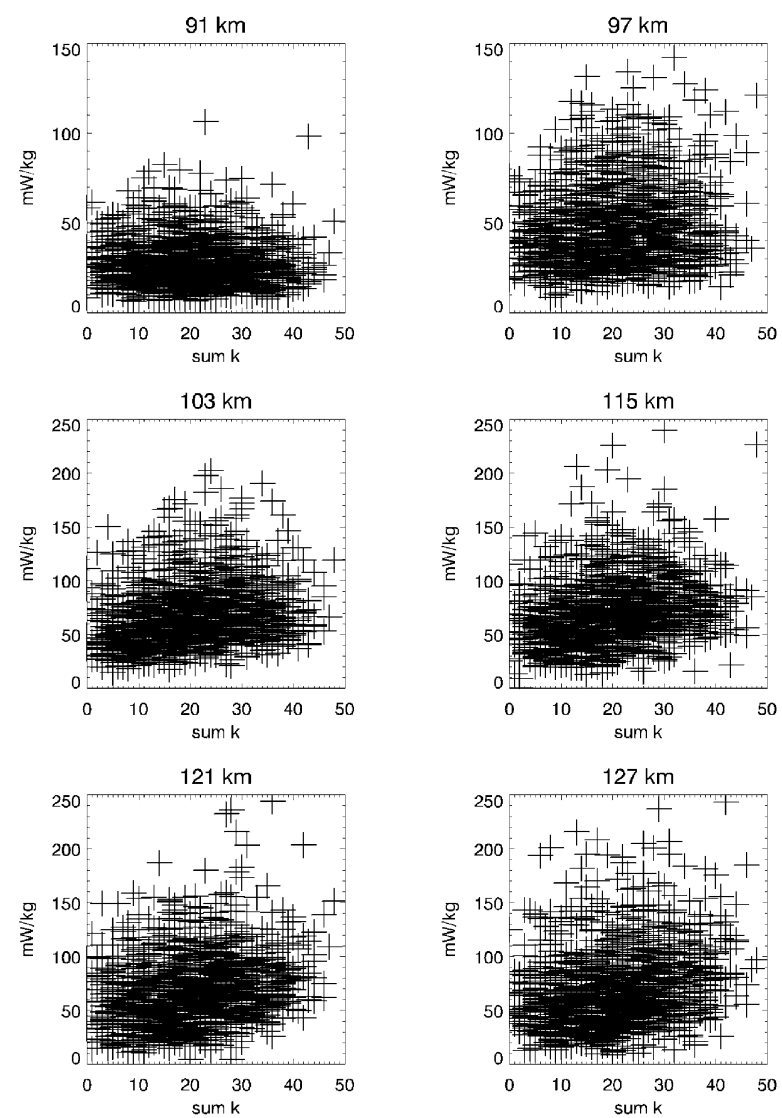

Fig. 3. Upper limit for day-averages of signal fading time expressed as energy dissipation rate, $\epsilon^{\prime}$, as a function of $\Sigma k$ (the sum of the 3 hourly local indices) for a variety of altitudes spanning the expected turbopause.

ing turbulence higher up. We shall illustrate correlation coefficients forthwith.

The next step is to search for evidence of correlation with individual three-hour $k$ indices. We produced scatter plots similar to Fig. 3 for 18-21 UT, 21-24 UT and the average of the 15-18 UT, 18-21 UT and 21-24 UT indices, the time intervals being selected to coincide with typical preferred times of auroral activity over Tromsø. Only the result of the comparison with the 21-24 UT $k$ index (henceforth denoted $k_{21-24}$ ) is shown here, this having the most pronounced grouping of the three intervals (Fig. 4). The results are not dissimilar from the $\Sigma k$ scatter plots but, of course, the discrete nature of the $k$ index itself becomes apparent. Figure 5 shows the result of performing a cross-correlation between $k_{21-24}$ and $\epsilon^{\prime}$ as a function of height. Nowhere is the correlation high. However, one must recall that only a weak correlation is expected, and, since geomagnetism has neither a prime influence on $\tau_{1 / 2}$ nor is it the prime driving force of neutral turbulence, finding a high correlation here would almost certainly suggest an erroneous result. It is interesting to note that there is consistency between altitudes and this indeed gives credence to the existence of a weak dependence. There is a minimum at the nominal mesopause; whether this is a coincidence is open to conjecture. Also shown in the figure, for comparison, is the correlation at a lag of one day $\left(\epsilon^{\prime}\right.$ lagging $\left.k_{21-24}\right)$. Other lags, not shown here, exhibited negligible correlation coefficient. It is in- 

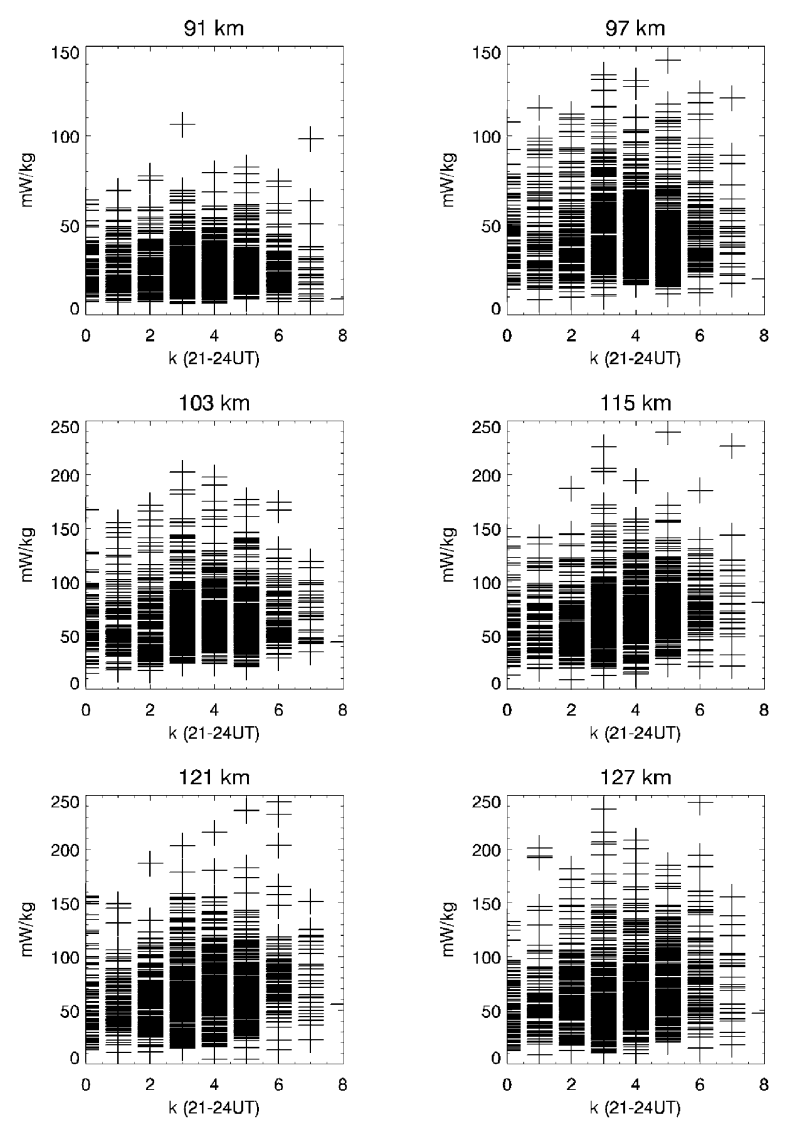

Fig. 4. As for Fig. 3 except that the 21-24 UT $k$ index has been used.

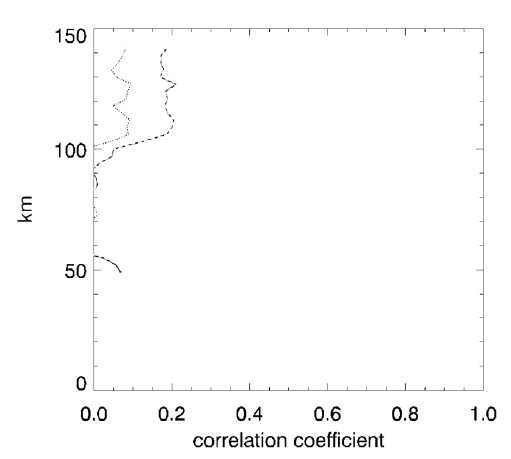

Fig. 5. Correlation coefficient as a function of altitude from the cross-correlation between day-averaged $\epsilon^{\prime}$ and corresponding 21-24 UT $k$ index (solid line). The dotted line indicates the correlation for $\epsilon^{\prime}$ lagging one day behind $k$.

teresting to note that above $105 \mathrm{~km}$ the coefficient remains fairly constant. This could be indicative of electromagnetic perturbation and/or dissipation of the scatterers in the ionospheric E-region, where neutral turbulence is inhibited.

As an alternative to attempting linear regressions to the $\epsilon^{\prime}$ versus $k_{21-24}$ scatter plots, we have chosen to bin the $\epsilon^{\prime}$ data and form averages of each bin, a natural approach since $k_{21-24}$ is already discrete (R. G. Roper, private communication). This is illustrated in Fig. 6, which shows bin-average $\epsilon^{\prime}$ height profiles as a function of $k_{21-24}$ bin. Making linear fits to the averages resulted in Fig. 7, the horizontal "error"

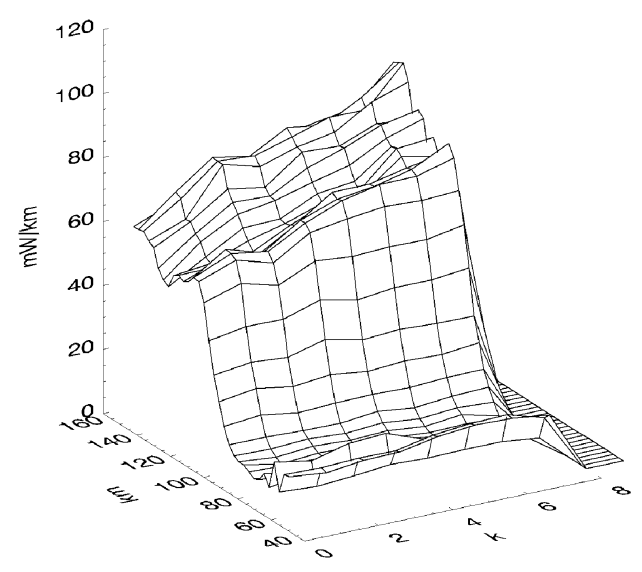

Fig. 6. For each value of the 21-24 UT $k$ index (i.e. 0-9), averages of $\epsilon^{\prime}$ have been formed (i.e. averages of the "columns" in Fig. 4). These are shown as a function of both $k$ and altitude.

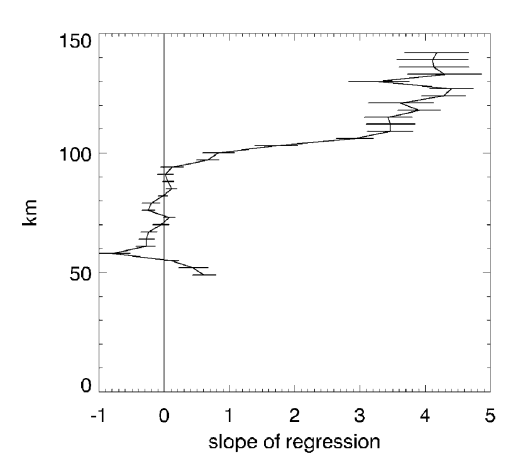

Fig. 7. Height dependence of slopes of linear regressions between the 21-24 UT $k$ index and the averages of the binned $\epsilon^{\prime}$ at each altitude. The horizontal bars indicate the 1-sigma uncertainties. These uncertainties should be interpreted as a combination variability of the primary turbulent generation and the geomagnetic effects.

bars indicating the 1-sigma uncertainty in slope. The uncertainties are substantial and exceed the magnitude of the slope itself at most altitudes below $90 \mathrm{~km}$. Between 90 and $120 \mathrm{~km}$, however, although the uncertainties are large, there is a systematic tendency towards a positive gradient. Yet again, it must be remembered that these uncertainties reflect the widths of the $\epsilon^{\prime}$ distributions in each $k_{21-24}$ bin, and this width in turn is a consequence of the other, more important mechanisms reducing signal fading time.

Since the mean time of a day average of $\epsilon^{\prime}$ precedes that of a $k_{21-24}$, and the $k_{21-24}$-effect is presumed to cause the $\epsilon^{\prime}$ perturbation, we have repeated the above approach for average $\epsilon^{\prime}$ for the 21-24 UT period each day. The results are shown in Fig. 8 (corresponding to Fig. 6) and Fig. 9 (corresponding to Fig. 7). This selective comparison yields the same basic result as when taking the entire day for $\epsilon^{\prime}$ averaging. While this approach does not detract from our conclusion, the correlation seems less clear; we do not know why this should be so: the cause could be a manifestation of poorer statistics, or a larger proportion of MF signals being affected by group retardation. At the same time, it is difficult to anticipate both the response time of the neutral atmosphere and the typical time of, for example, maximum 


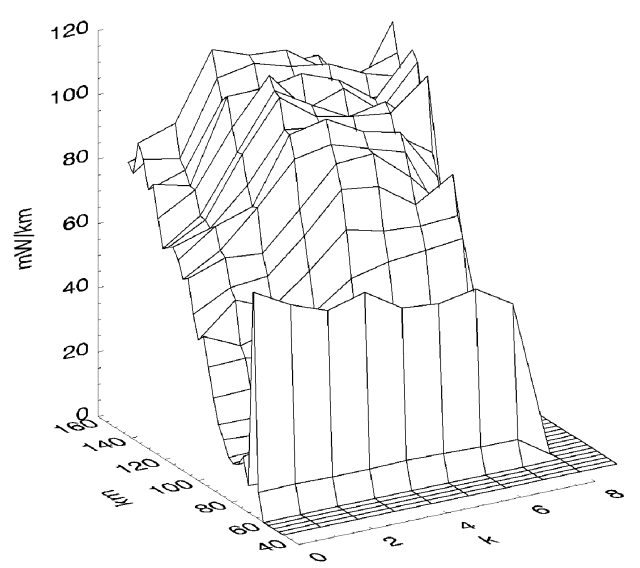

Fig. 8. As for Fig. 6, but with $\epsilon^{\prime}$ averaged over 21-24 UT, rather than the whole day.

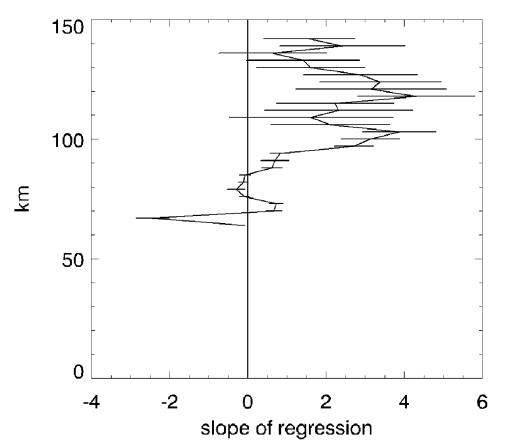

Fig. 9. As for Fig. 7, but with $\epsilon^{\prime}$ averaged over 21-24 UT, rather than the whole day.

ion drag. It is conceivable that our choice of the 21-24 UT period was unfortunate. Similarly we cannot assume that ion drag is indeed responsible for destabilising the neutral flow. $\mathrm{Xu}$ et al. (2001) have, for example, investigated photochemical effects on gravity waves, an effect enhanced by atomic oxygen, this constituent in turn being influenced by auroral precipitation, hydroxyl and turbulent mixing in a complex feedback process. A multitude of selection schemes involving both time and height combined with various lags could be envisaged but largely based upon a priori assumptions as to the mechanism underlying the correlation; therefore, we shall not confuse the issue further here.

By examining yet another parameter, one reflecting the dynamics responsible for turbulent generation, it might be possible to remove these effects from the time series making the geomagnetic influence easier to isolate. To do this, partial correlation analyses between $\epsilon^{\prime}$ versus $k_{21-24}$ were performed in which each of the corresponding horizontal zonal, meridional and total wind variations were specified as the variations to ignore. In each case, the correlation coefficient profile was unaffected. In many ways this was not surprising because the FCA winds are known not to correlate strongly (Manson and Meek, 1987) with the FCA fading times, this being used as a control that signal fading is indeed due to turbulence and not due to scatterers being removed from the radar field of view by the horizontal wind. A similar experi- ment was performed by including low altitude $\epsilon^{\prime}$ (this would be less affected by geomagnetic effects than $\epsilon^{\prime}$ near the electrojet) as the variability to ignore in the cross-correlation. Again, the results were unaffected. The results of the partial correlations are not shown here. It would be possible to derive other parameters designed to reflect the non-solar and non-geomagnetic influence on $\tau_{1 / 2}$ or turbulence generation, however it becomes increasingly difficult to be certain that these are truly independent.

\section{Discussion}

The main impression one gets from the preceding figures is that any correlation between signal fading time reduction and external forcing is very weak and that the correlation coefficients are none too convincing. As stated earlier, though, the convective and dynamic instabilities that are the sources of any turbulence are primarily generated by shears and gravity waves driven by larger scale circulation, and the effects we are searching for are likely to be minor perturbations, at least, below the turbopause. It is regrettable that the search for a parameterisation of the primary turbulence generation proved fruitless, because subtracting its effect would almost certainly have revealed the secondary solar and geomagnetic effects unambiguously. On the other hand, by removing other dependencies that may be significant, as Fig. 8 maybe suggests, the effect of the geomagnetic field is shown to be very small although still present.

Due to the lifetime of auroral disturbances, it would be unreasonable to apply smoothing to the $\tau_{1 / 2}$ 's. Similarly filtering out any longer periods is a dangerous practice since solar forcing is almost always an underlying mechanism. Quite apart from the observations of Danilov et al. (1979) leading us to anticipate geomagnetic influence on turbulence, note that this study entails 1380 profiles (although data does not always exist at each altitude) and that consistency is seen between adjacent altitudes. Thus, although correlation coefficients are not high, we feel the evidence for geomagnetic influence is strong. Nevertheless, there are a number of caveats and assumptions that need to be clarified.

MF radar returns are liable to be subject to group delay of the radio wave in the ionised medium. The heights we quote here are all virtual heights and the true heights may well be lower, although this is a direct function of the degree of ionisation. Furthermore, when ionisation is considerable, total reflection of the radio wave may occur such that echoes only appear to arrive from altitudes above the total reflection height. On the one hand, out of the 1380 profiles, a rather small number can be expected to occur when aurora was in the field of view, such that the total reflection and group delay problems do not apply on the majority of occasions, at least up to $120 \mathrm{~km}$. On the other hand, the very geomagnetic or solar flux related effects we are attempting to search for are most likely to be associated with particle precipitation and therefore the high degrees of ionisation that cause these group delay limitations. In order to investigate the problem, we implemented the screening method for the MF data used by Nozawa et al. (2001). In this method, the height of maximum echo amplitude above $91 \mathrm{~km}$ is identified, and all signal from above that height is deemed to be suspect 


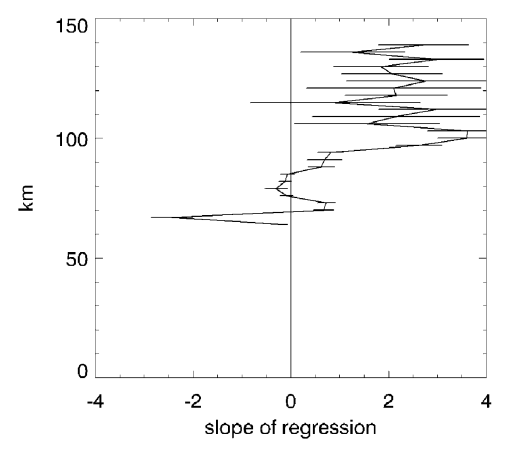

Fig. 10. As for Fig. 9, but using the drastic $\epsilon^{\prime}$ screening method described by Nozawa et al. (2001) (see text).

due to large group delay or total reflection. One cannot assume, a priori, that useful data do not exist from above this height, and therefore the method is drastic, to say the least. Nevertheless, even with the 21-24 UT selection, we obtained over 200 data points at the highest altitudes (a yield of $14 \%$ ). Furthermore, the result (Fig. 10) was similar to the comparisons using unscreened $\epsilon^{\prime}$.

The derivation of $\epsilon^{\prime}$ in itself involves assumptions, although well documented by Hocking (1999). As an example, a model value of the Brunt-Väisälä period is used as a time scale and this cannot a priori be expected to be independent of solar heating. Even though heating may be most pronounced in the stratosphere due to UV absorption, the modified gravity wave flux will cause the quiescent turbulent energy dissipation rate and therefore energy budget to change in the mesosphere, thus perturbing the Brunt-Väisälä period. Quite apart from the assumptions inherent in estimation of $\epsilon^{\prime}$, it should not be forgotten that this parameter is, after all, only a way of expressing the fading time. Moreover, how well $\epsilon^{\prime}$ represents the actual turbulent energy dissipation rate $\epsilon$, even in the mesosphere, is open to debate, as discussed earlier.

Danilov et al. (1979) determined the turbopause height by an in situ method and then compared a somewhat limited dataset with the local $a_{k}$ index, the linear equivalent of $k$. Not shown here, we have examined scatter plots of $\epsilon^{\prime}$ versus $a_{k}$ indices corresponding to the various $k$ index combinations described earlier; it was felt that the latter gave clearer groupings of points. In addition to comparison with the $a_{k}$ index, Danilov et al. (1979) noted a seasonal variation in turbopause height, again without an explicit explanation. Thus, it might be tempting to filter the $\epsilon$ 's in order to remove, say periods greater than 6 months. However, we return to the question as to what is influencing this periodicity, and, inevitably, we identify solar forcing.

So even though the observational evidence for geomagnetic influence on turbulence is somewhat tenuous, it is supported by the quite independent study of Danilov et al. (1979) and by the theoretical prediction of Hall (2001a). The $k$ index directly reflects the quasi logarithm of the geomagnetic perturbation (Jacobs, 1989, and specifically http://www.tgo.uit.no/knum/], and therefore the current and, in turn, the plasma velocity. We have seen that sufficiently high velocities exist within restricted height regimes to cause strong local ion drag (Hall and Aso, 2000) and that these height regimes coincide with the turbopause. Previously Hall et al. (1998) have attempted to locate the turbopause and have indeed identified the same annual height variation as Danilov et al. (1979); however, in order to avoid introducing too many assumptions, we have restricted ourselves to $\tau_{1 / 2}$ in this study.

Turbulent intensity, parameterised by $\epsilon^{\prime}$ at the top of the homosphere, in determining the height of the turbopause, is of interest because it indicates mixing efficiency in the lower thermosphere. The higher the turbopause, the higher up turbulence may re-distribute species such as methane (important for water chemistry). Similarly, molecular oxygen is dissociated in the ionosphere; if the turbopause is located within the atomic oxygen production regime, the atomic oxygen may then be transported downwards enabling it to affect the ozone chemistry in the upper mesosphere. Another consequence of this transport can be the destabilisation of gravity waves, as mentioned earlier $(\mathrm{Xu}$ et al., 2001). Thus, geomagnetic disturbances originating in electrodynamics may be capable of affecting vertical transport of species at high latitude, which in turn dictate phenomena such as noctilucent clouds and affect, for example, ozone content.

\section{Conclusions}

Independent observational and theoretical studies suggest that strong perturbations in the geomagnetic field are capable of reducing radar echo fading times and therefore enhancing neutral air turbulence in the upper mesosphere and possibly lower thermosphere. In particular, this geomagnetically enhanced turbulence, occurring at the top of the homosphere may cause a lifting of the turbopause, thus affecting the height distribution of minor constituents. To investigate this, a four-year sequence of estimates of signal fading time expressed as turbulent energy dissipation rate, $\epsilon^{\prime}$, by the Ramfjordmoen (Tromsø) MF radar have been compared with corresponding solar flux parameters and with geomagnetic indices, both planetary and local. Although a strong relationship should not be expected since the short term solar and geomagnetic forcing are very much secondary mechanisms for destabilisation of the neutral flow, a weak correlation indeed exists between the local geomagnetic field and reduction in signal fading time in the height regime 100-120 $\mathrm{km}$ at $69^{\circ} \mathrm{N} 19^{\circ} \mathrm{E}$.

Acknowledgments. Thanks go to the technical staff of the Troms $\varnothing$ Geophysical Observatory for maintenance of the Tromsø MF radar. We acknowledge the use of $10.7 \mathrm{~cm}$ flux from the Herzberg Institute of Astrophysics, Penticton, Canada.

\section{References}

Briggs, B. H., The analysis of spaced sensor records by correlation techniques, Handb. MAP, 13, 166-186, 1984.

Danilov, A. D., U. A. Kalgin, and A. A. Pokhunov, Variation of the mesopause level in polar regions, Space Res. XIX, 83, 173-176, 1979.

Gavrilov, N. M., A. H. Manson, and C. E. Meek, Climatological monthly characteristics of middle atmosphere gravity waves (10 min to $10 \mathrm{~h}$ ) during 1979-1993 at Saskatoon, Ann. Geophys., 13, 285-295, 1995.

Hall, C. M., An analogy to the Reynolds number for the neutral gas component of a weak plasma, Ann. Geophys., 19, 1-4, 2001 a.

Hall, C. M., The Ramfjormoen MF radar $\left(69^{\circ} \mathrm{N}, 19^{\circ} \mathrm{E}\right)$ : Application development 1990-2000, J. Atmos. Solar Terr. Phys., 63, 171-179, 2001 b. 
Hall, C. M. and T. Aso, Identification of possible ion-drag induced neutral instability in the lower thermosphere over Svalbard, Earth Planets Space, 52, 639-643, 2000.

Hall, C. M., A. H. Manson, and C. E. Meek, Measurements of the arctic turbopause, Ann. Geophys., 16, 342-345, 1998.

Hocke, K. and K. Igarashi, 24- and 12-h oscillations during a time of strong eastward wind in the upper mesosphere at $31^{\circ}$ and $45^{\circ} \mathrm{N}$, J. Atmos. Solar Terr. Phys., 60, 1071-1079, 1998.

Hocking, W. K., The dynamical parameters of turbulence theory as they apply to middle atmosphere studies, Earth Planets Space, 51, 525-541, 1999.

Holdsworth, D. A., R. A. Vincent, and I. M. Reid, Mesospheric turbulent velocity estimation using the Buckland Park MF radar, Ann. Geophys., 2001 (in press).

Jacobs, J. A., Geomagnetism Volume 3, 533 pp., Academic Press, London, 1989.

Luo, Y., A. H. Manson, C. E. Meek, K. Igarashi, and Ch. Jacobi, A quasi 27-day oscillation in the mesospheric and lower thermospheric wind: Observations in Europe, Japan and Canada, Report \#1 Atmospheric Dynamics Group, University of Saskatchewan, Canada, 31 pp., 2000.

Manson, A. H. and C. E. Meek, Comparisons between neutral winds near
$100 \mathrm{~km}$ at Saskatoon $\left(52^{\circ} \mathrm{N}, 107^{\circ} \mathrm{W}, L=4.4\right)$ and variations in the geomagnetic field (1979-1983), Ann. Geophys., 4, 281-286, 1986.

Manson, A. H. and C. E. Meek, Small-scale features in the middle atmosphere wind field at Saskatoon, Canada $\left(52^{\circ} \mathrm{N}, 107^{\circ} \mathrm{W}\right)$ : An analysis of MF radar data with rocket comparisons, J. Atmos. Sci., 44, 3661-3672, 1987.

Meek, C. E., An efficient method for analysing ionospheric drifts data, $J$. Atmos. Terr. Phys., 42, 835-839, 1980.

Meek, C. E., Triangle size effect in spaced antenna wind measurements, Radio Sci., 25, 641-648, 1990.

Nozawa, S., A. Brekke, A. H. Manson, C. M. Hall, C. E. Meek, K. Morise, S. Oyama, K. Dobashi, and R. Fujii, A comparison study of the auroral E region neutral winds derived by the EISCAT UHF radar and the Troms $\varnothing$ MF radar, J. Geophys. Res., 2001 (in press).

Xu, J., A. K. Smith, and G. P. Brasseur, Conditions for the photochemical destabilization of gravity waves in the mesopause region, J. Atmos. Solar Terr. Phys., 63, 1821-1829, 2001.

C. M. Hall (e-mail: chris.hall@tgo.uit.no), S. Nozawa, C. E. Meek, and A. H. Manson 